\title{
Image Organization and Navigation Strategies for a Radiological Work Station
}

\author{
Etienne Roger, Morris Goldberg, and Richard F. Dillon
}

\begin{abstract}
One can distinguish two distinct preliminary phases in the "analog" radiographs reading process. The first phase deals with the film selection and placement on viewboxes (image organization), and the second with the actual film reading (involving image navigation, ie, the means for accessing the individual images). Our multimedia radiological work station will replace the view boxes with a low-resolution control monitor and a single high-resolution image monitor for viewing the radiographic images of the patient. These are organized by body area and examination view, and are associated with a sketch of a human body on the control monitor. This "anthropomorphic" organization of the images helps the radiologist create a mental picture of the entire patient radiographic file. We expect that together with the requisition information, a proper representation of the file will improve the search for relevant radiographic images. The work station supports image navigation by means of a "soft" representation of the physical viewboxes. In the same manner that radiographic films can be placed onto physical viewboxes, image labels can be dragged with the mouse onto soft viewboxes embedded on the image monitor. Evaluation of results from tests conducted with 15 physicians are presented.

(C) 1989 by W.B. Saunders Company.
\end{abstract}

KEY WORDS: PACS, user interface, user testing, radiologic work station.

$\mathbf{P}$ ICTURE ARCHIVING and communication systems (PACS) provide storage for digital pictures or images and means for retrieving and transmitting them over electronic media. ${ }^{1}$ The four main components of a PACS are an image storage subsystem (eg, optical disk jukebox), a communication medium (eg, fiber optic network), image acquisition devices, and endpoint work stations (terminals) for retrieving images.

Growing research in the field of PACS applied to radiology highlights the need for adequate digital radiograph viewing work stations. ${ }^{2-8}$ The main goal is to provide the necessary information (patient file, radiographs) and facilities for radiologists to generate appropriate medical reports. The literature abounds with studies of preferences and requirements for viewing work stations. The simplicity of usage and fast access to the images certainly are desired features. ${ }^{9}$ Rogers et $\mathrm{al}^{6}$ recommend a facility to compare corresponding views between different examinations (to analyze progression of anomalies through time, for example). The ability to maintain the spatial relationship between images, as with view boxes, is also recommended by Rogers et al. ${ }^{6}$ and Kasday. ${ }^{4}$ Film placement on viewboxes is also subject to personal preferences among radiologists. For example, hand views may be placed with fingertips pointing up or down; lateral views of the chest may be seen with pectorals facing left or right (D. Wittingham, personal communications, 1988). Care must be taken, though, when analyzing viewing work-station requirements as most studies were made using computed tomographic (CT) scan images; their results may not be generalized to other image modalities (eg, digitized projection roentgenographic films).

Various work station design strategies also have been reported. The CommView ${ }^{4}$ system (AT\&T) is equipped with a keyboard, a mouse, and three monitors aligned horizontally. The leftmost screen is reserved for text and therefore has the keyboard; the others are used for displaying the images. Initially, a patient file is presented as a stack of folders on one monitor. Images or reports are then accessed by bringing the relevant folders to the top of the stack or to any other monitor. A single mouse pointer covers all three monitors and is used for selecting folders, and flipping through pages of folders.

The Arizona Viewing Console ${ }^{5,8}$ (University of Arizona) is equipped with a keyboard, trackball, knobs, and three monitors (a color command monitor and two monochrome high-resolution image display monitors). A patient file is selected by typing the patient's name. Then, from an image directory displayed on the command monitor, images are selected and placed anywhere on

From the Medical Communications Research Centre, Department of Electrical Engineering, University of Ottawa, Ontario, Canada.

Funded by the Telecommunications Research Institute of Ontario, Bell Canada, and BNR.

Address reprint requests to E. Roger, APSIS, BP 177 , 38244 Meylan cedex, France.

(c) 1989 by W.B. Saunders Company.

0897-1889/89/0204-0006\$03.00/0 
either display monitor. Image processing and manipulation operations affect only one image at a time, the one designated as the active image. All three monitors are fitted with pressuresensitive screens that allow image selection and activation to be performed by a simple finger touch.

The Philips' MarView work station (Philips, Shelton, CT) proposed by Schuttenbeld and Romeny ${ }^{7}$ is equipped with a mouse and four monitors. One monitor, the "desk part" of the visual work space, displays administrative and system information. The other three, the "lightbox part" of the visual work space, are used as electronic light boxes for image display. The three electronic light boxes are represented on the desk-part by three horizontally adjacent windows. Images are displayed on an image monitor by placing the appropriate image icon in the corresponding light-box window.

A common characteristic of these systems is the constraint imposed by the physical image viewing surface on the spatial arrangement of the images: the number and layout of light-box windows correspond to the number of physical monitors. $^{10,11}$ The traditional arrangement of view boxes in radiologists' offices (two rows of four view boxes each) also represents a limited viewing surface. If more films need to be viewed than can be displayed at once, then a radiologist has two options: to manually exchange films or to use an "alternator" device. ${ }^{12}$ The alternator is a mechanical, conveyor belt-type of device that provides serial access to a store of images. Hence, for an electronic image work station, some mechanism must be provided for accessing the images of sets that exceed the display capacity of its physical viewing surface.

There are two distinct preliminary phases in the "analog" reading process. The first phase deals with the film selection and placement on the view boxes (a process we call "image organization"), and the second with accessing these images by virtue of their respective locations on the bidimensional view-box surface (a process we call "image navigation"). In our work station, the list of radiographic images of a patient is organized (classified, sorted) and presented on a low-resolution control monitor $(640 \times$ $400 \times 1)$ from which the images to be viewed on an adjunct high-resolution image monitor $1,024 \times 1,280 \times 8)$ are selected.
Image navigation is supported in a manner similar to the analog world's view boxes through the use of a "soft" view-box surface. The important difference is that we use only a single monitor (the image monitor) for viewing the radiographic images. The image monitor essentially is a window that moves over the soft view-box surface. The digital view-box surface thus effectively provides a total image work space of comparable size to the analog one. The user interface designed for the work station addresses the particular issues of image organization and navigation.

In the next section, the design of the image organization and navigation strategies is presented. The implementation of the above-mentioned strategies using an object-oriented programming language also will be described. Finally, we will present some results of a field evaluation conducted with 15 physicians.

\section{IMAGE ORGANIZATION AND NAVIGATION DESIGN}

\section{Radiograph Reading Process}

Radiologists read radiographic images of patients and report their findings to the referring physicians. The entire patient radiograph file is presented to the radiologist together with a requisition form from the physician which states the reason for ordering the radiographic examination. All the radiographs of a patient are normally stored in one large envelope or "master envelope," within which smaller envelopes or jackets may be found. In general, each jacket corresponds to one examination. The requisition statement is included in the examination jacket and can be as succinct as "? Fract LT Prox Tib" ("Is the left proximal tibia fractured?"). To answer the requisition statement it is often necessary to inspect previous examinations. Initially, the radiologist places all the films of the latest examination on the view boxes. If comparison with previous examinations is required, a search for relevant films begins. First, the radiologist may consult the list of the examinations contained in the master envelope. Second, the radiologist may consult the requisition forms of the older examinations. Third, he or she may read the transcribed reports from these older examinations. Fourth, the radiologist may partially pull out films from their examination jackets, placing on the view boxes those required for comparison 
with the present examination. At this point the set of relevant radiographs has been arranged in some manner on the view boxes. The radiologist then reads the films and reports any findings in a general report which also responds to the requisition statement.

Two distinct preliminary phases can be distinguished in the analog reading process. The first phase deals with the film selection and placement on the view boxes (image organization), and the second with the actual film reading (involving image navigation). We will now analyze the present analog way of handling radiographic image organization and navigation in more detail. This will be followed by a proposed "digital" replacement.

\section{Image Organization and Navigation \\ Image Organization}

Radiographs are normally contained in a large master envelope (Fig 1), within which may be found smaller envelopes or jackets (Fig 2). On the master envelope is found the patient's full name along with an unique identification number, and an index listing the examinations contained therein. An examination is a set of radiographs (average, four) taken within a relatively short time span and in response to a specific requisition. The set of radiographs is collected in a jacket which is inserted into the master envelope. Each jacket also bears the patient's name and unique identification number in addition to an examination number. The requisition form is pasted onto this jacket. Examinations are listed and stored chronologically; each new examination is listed at the bottom of the index and inserted at the front of the master envelope. Within a jacket no specific order prevails for the individual images.

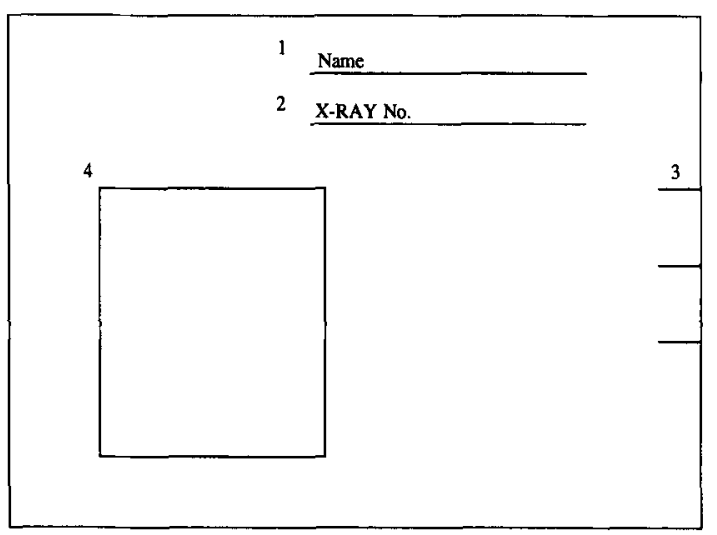

Fig 2. Examination envelope (Ottawa Civic Hospital). (1) Patient first and last name; (2) patient five-digit examination number; (3) patient unique identification number; (4) requisition form.

A number of problems characterize the analog image organization strategy. First, although the most recent examination jacket is usually found at the front of the master envelope, chronological ordering of the others is not guaranteed. Moreover, it is not uncommon to find misplaced radiographs of a particular examination in some other examination's jacket or even on occasion in some other patient's master envelope. Misplaced radiographs cause time-consuming searches and require extraneous envelope maintenance on the part of the radiologist. Second, the organization is static and does not support any reorganization according to other criteria or user preferences. Indeed, during test sessions, radiologists suggested other methods of sorting examinations (other than chronological). These were (1) organization by body area (eg, hand); (2) organization by organ system (eg, abdomen); and (3) organization by clinical problem (eg, pneumonia).

Within an examination containing many views, it also may be appropriate to sort the images

Fig 1. Master envelope (Ottawa Civic Hospital). (1) Patient first and last name; (2) patient seven-digit unique identification number; (3) least significant digits (three) of the unique identification number; (4) colorcoded four most significant digits of the identification number; (5) examination reports pocket: (6) list of examinations contained in the envelope.

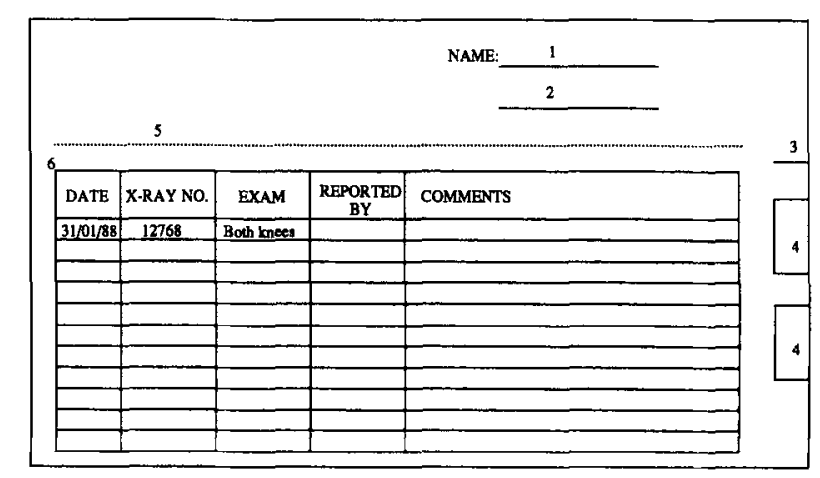


chronologically (eg, for times series) or in some specific order according to the examination at hand (eg, for anterior-posterior [AP] and lateral chest pair of views; AP may always be listed first, then lateral). Another organizational problem is that film manipulation is cumbersome and lengthy. ${ }^{6}$ During the reading process, many films, possibly from different examinations, are pulled out of their envelopes; only some of these will be placed on the view boxes. A study performed with CT-scan films showed that about $35 \%$ of a radiologist's time is spent reviewing the patient's history and sorting and placing films on view boxes. ${ }^{6}$ After report dictation, the radiologist has to sort these films and place them back into their proper envelopes.

\section{Image Navigation}

Radiographic films are read by placing them between a light source and the observer. View boxes are semitransparent back-lit surfaces (14 in wide $\times 17$ in high) that gave a fairly uniform source of light for the reading of films placed on their surface. View boxes are normally found in three different configurations: a single view box; a pair of adjacent view boxes; or a set of eight (two rows of four boxes, one above the other). The latter configuration is the most prevalent form in radiology. Each view box can be illuminated independently from a set of switches. Normally, radiologists light only the view boxes that receive a film; the others are kept turned off to avoid creating distracting light sources. The view-box case enables radiologists to quickly refer to any image by a simple eye or head movement.

By arranging the films of interest on the viewbox case, radiologists achieve a well-structured bidimensional indexing of these images. ${ }^{11}$ Some radiologists use the view boxes to "reconstruct" the subject's body from the available views (D.
Wittingham, personal communications, 1988). However, when comparing a pair of images, radiologists place images of interest either in adjacent view boxes or one image directly above the other, as large eye movements between distant view boxes can be distracting. Radiologists also arrange the films of interest on the view-box surface according to their preferences. For example, assuming four pairs of chest roentgenograms (an AP and a lateral view for each pair), two possible arrangements are (1) vertically, all APs on one row and all laterals on the other, or (2) horizontally, an AP and a lateral side by side (left to right), two pairs per row.

\section{Proposed Solution}

Our image organization strategy attempts to solve some of the purely mechanical problems related to film handling and provide a flexible presentation of the corresponding images. Our image navigation strategy mimics the organizational property of view boxes by providing a bidimensional surface for image indexing.

\section{Image Organization}

The proposed digital image organization strategy is based on an "anthropomorphic" organization (Fig 3). The center of the control monitor is occupied by the sketch of a human body around which labels of images referring to body areas are grouped. This graphic index of images provides an overview of the image contents of the patient folder. The list of image labels related to a body area is found by selecting the body area label (Fig 4). The image labels give the following information: (1) name of body area or procedure (eg, chest or cervical spine-myelogram); (2) name of view (eg, lateral or AP); (3) time when the film was taken (eg, 10:30); and (4) examination number (eg, 64220).

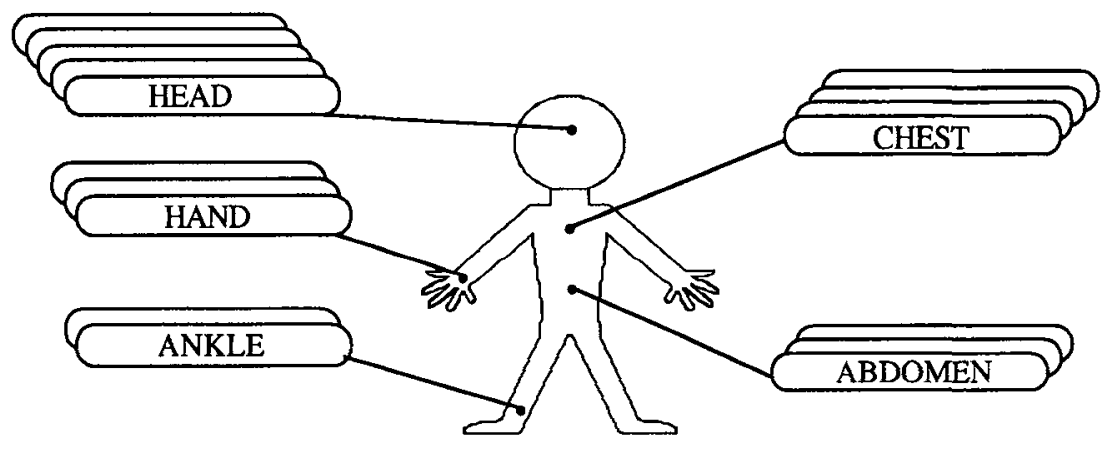

Fig 3. Anthropomorphic image organization strategy. 


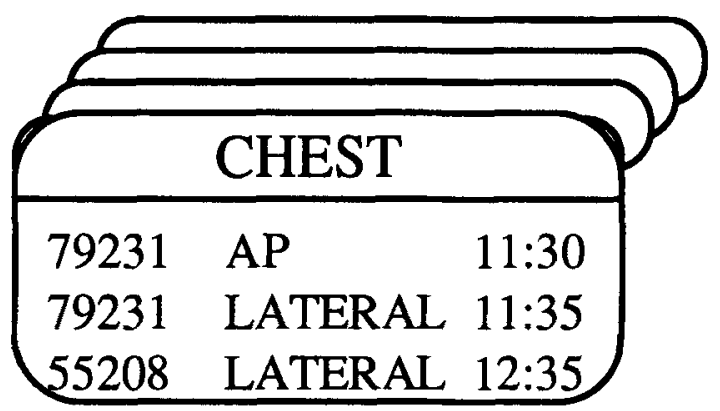

Fig 4. Body-area list of views.

Labels can be sorted according to the user's preferences (eg, chronologically or alphabetically by view). An image is displayed on the image monitor by selecting its corresponding label. The necessity for selecting a body area in order to access the related views, rather than permanently displaying them, is justified by the need to minimize screen clutter (information density). ${ }^{13}$ The thickness of the stack of buttons (round-cornered rectangles) beneath each body area label provides an indication of the number of images related to that area. As examinations usually comprise a standard number of views, this enables radiologists to rapidly locate areas for which the number of views differs from the norm. For example, this could be an indication that difficulties were encountered during the examination due to the patient's severe condition.

The anthropomorphic organization of the images helps the radiologist create a mental picture of the entire radiograph file. Together with the requisition statement, a proper mental representation improves radiograph database search by constraining it to the relevance of the case under examination. This organization strategy supports different ways of sorting and classifying the images. Also, film manipulation and the need for sorting films after reporting have been eliminated. This in turn eliminates the problem of misplaced radiographs.

\section{Image Navigation}

Because radiologists rely on some form of spatial arrangement of the films in the analog world, a similar mechanism must be provided in the digital world. Hence, the image navigation strategy is based on a soft representation of the physical view boxes (Fig 5). This strategy essentially is a Spatial Data Management System (SDMS). ${ }^{11}$ Information in an SDMS is represented by icons arranged in some data space. Icons can be used as a navigational aid through the database. ${ }^{10}$ In general, an icon has two representations: a visual (physical) representation and an abstract (logical) representation. An icon is visually represented by some sketch that reflects the attributes of the related database entity. The abstract representation of an icon conveys the information that identifies and locates the related database entity.

The soft view-box surface constitutes a bidimensional space on which the user arranges radiographs represented by icons. The image

Fig 5. Soft view-box-based image navigation strategy.

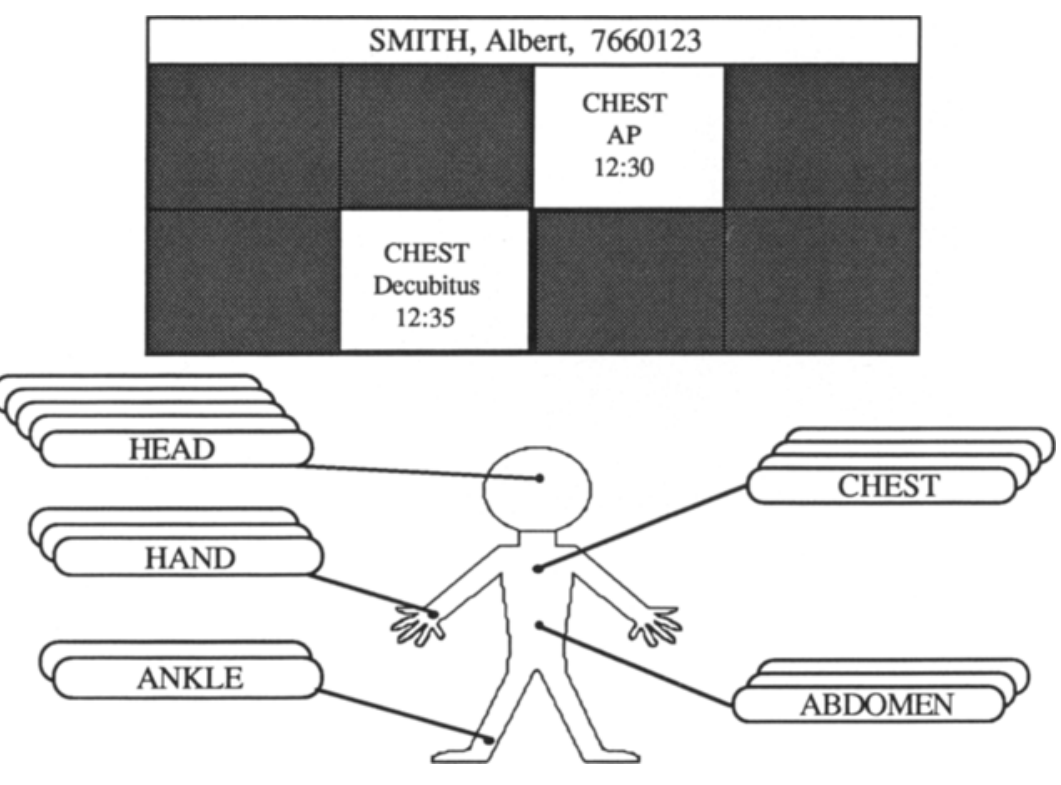


icon's visual representation is composed of alphanumeric characters and lists the name of the body area of the related radiograph, the name of the view and time of day it was taken. The visual representation also could be replaced by a reduced resolution view of the original image. The image icon's logical representation consists of a data structure (which will be discussed later) that contains the above mentioned data and storage information for radiograph retrieval.

In the same manner that radiographic films can be placed onto real view boxes, image labels can be dragged with the mouse onto the soft view boxes for later viewing. Image icon placement on the view boxes also can be rearranged by dragging icons from view box to view box. Users directly manipulate radiographs through their iconic representations as they would their real counterparts, films. An image is displayed on the image monitor by selecting its corresponding icon. To avoid disturbing the actual film-reading process, images can also be selected for display from a replica of the view boxes that are embedded on the image monitor (Fig 6). Because a single pointing device covers the entire visual work space, the cursor has to be moved over to the image monitor for the user to access various image enhancement functions (Fig 6). Once the cursor is on the image monitor, the radiologist is able to concentrate on reading the radiographs and the associated report. To select an image that was placed previously on a view box, the embedded view boxes are popped up by clicking anywhere on the image monitor. Selecting an image then makes the pop-up view boxes disappear to make way for the new image. The use of embedded view boxes reduces the amount of interaction requested from the radiologist. In the following section we present the implementation of the image organization and navigation strategies.

\section{AN OBJECT-ORIENTED IMPLEMENTATION}

\section{Hardware}

The hardware configuration of the work station is shown on Fig 7. The core of the work station is a Compaq 386/20 microcomputer equipped with a large hard disk (for image

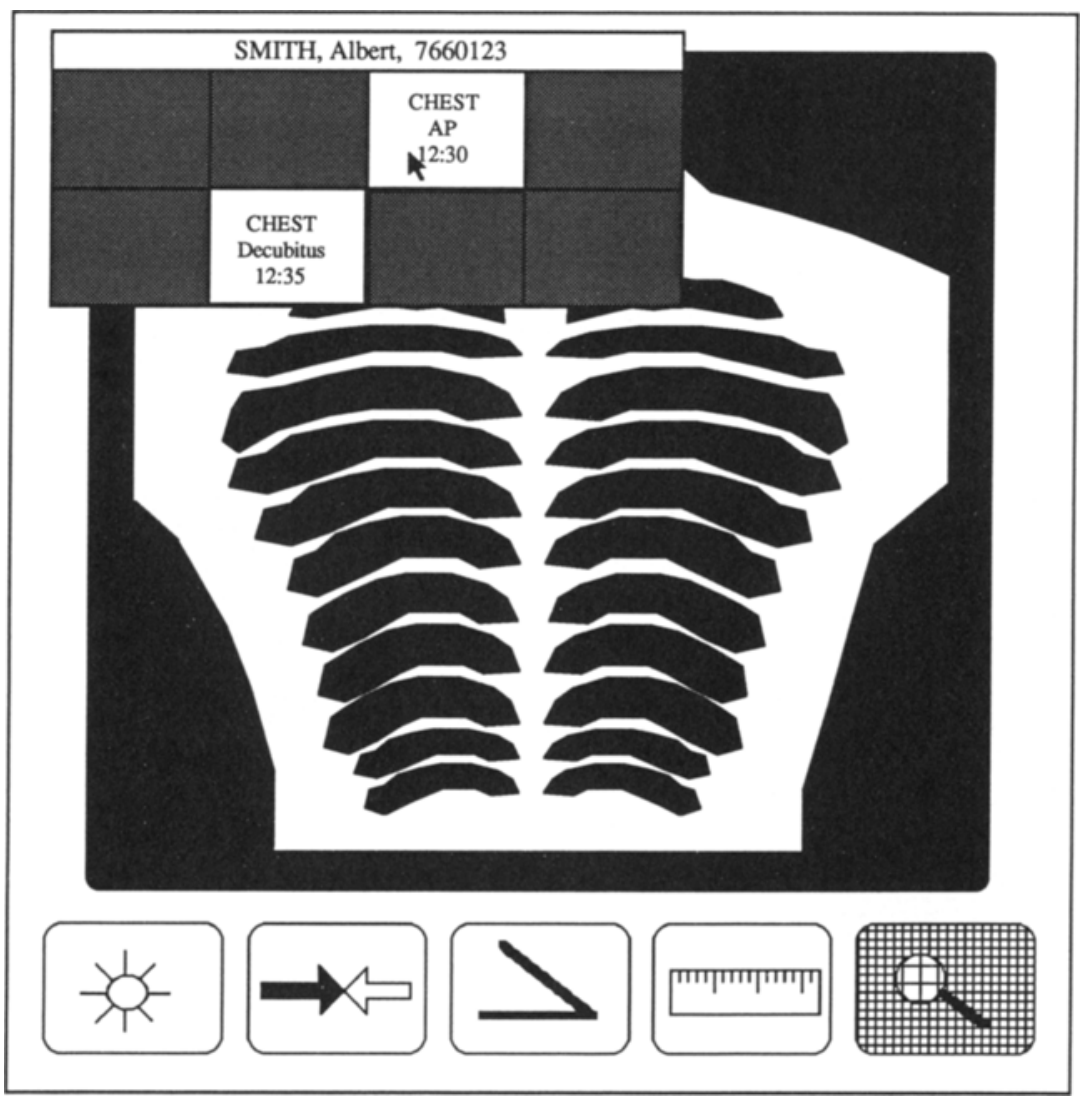

Fig 6. Image monitor with embedded view boxes. 


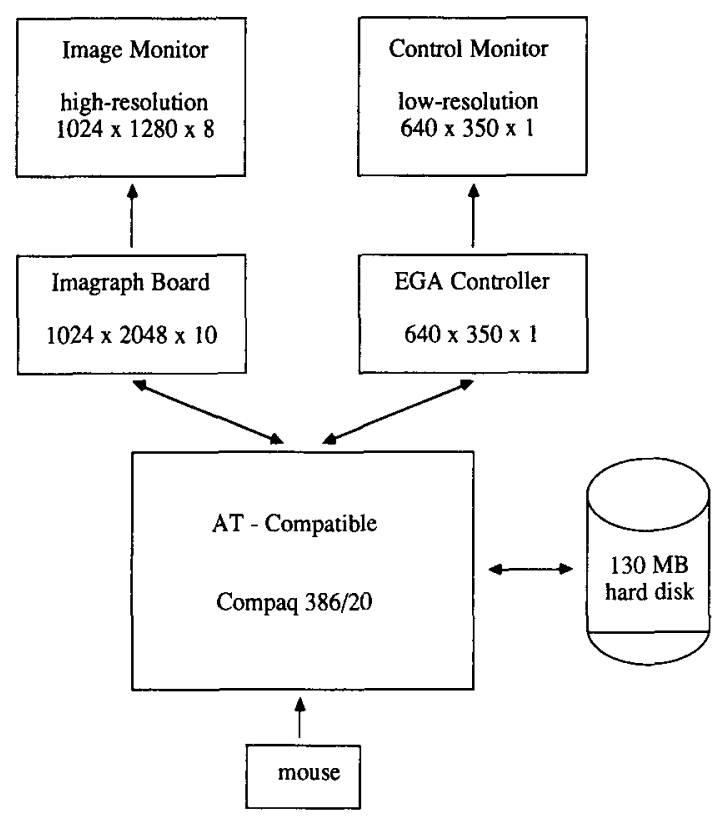

Fig 7. Work station hardware configuration.

storage). The visual work space is composed of two monitors: a low-resolution control monitor $(640 \times 350 \times 1)$ and a high-resolution image monitor $(1,024 \times 1,280 \times 8)$. A single mouse is used to reinforce the fact that the two monitors form a unified visual work space. The control monitor is driven by an enhanced graphic adapter (EGA; IBM, Danbury, CT) controller; the image monitor by an Imagraph (Woburn, MA) imaging board. The Imagraph board has a twoplane image memory; an eight-bit main-memory plane and a two-bit overlay plane. Both planes are $1,024 \times 2,048$ pixels although at most $1,024 \times 1,280$ pixels can be displayed at a time. Radiographs are displayed in the main-memory plane. The image processing icons, the embedded viewboxes, and the cursor (Fig 6) are displayed in the overlay plane.

\section{Software}

The user communicates with the system through a collection of active objects represented on the display monitors (eg, an image label on the control monitor). An object (or frame in artificial intelligence) is a named collection of information, similar to a record, to which are attached methods (procedures) which operate on the object's information. Objects send messages to other objects (or to themselves); upon reception of a message the receiver executes the appropri- ate method. Objects also may inherit methods and values from other objects. There are four main categories of objects; view, controller, model, and abstract data structure. A view object or pane takes care of framing, labeling, scrolling, bordering, and data presentation. The appearance of an object (to distinguish a selectable from an already selected object, for example) and its relations to others (as when an object is dragged) is defined at the level of the pane objects. Each pane object has a unique controller associated with it. A controller handles the stream of data from the input devices (keyboard and mouse) and performs window scheduling. The controller sends messages to its pane to take action according to the input events. A model is the underlying program or application that communicates with the user. The model is responsible for the creation of all panes and controllers it requires. It also is responsible for the communication and synchronization among these panes and their related abstract objects. A special "dependency" relationship ties a pane to its model so that an object then can broadcast messages to all its dependents at once. The abstract objects are data structures (eg, arrays, sets) used by the other objects.

The ImageFolder class of abstract objects (software developed by the authors) is central to the image organization and navigation strategies. An ImageFolder object (Fig 8) is created for each radiograph of a patient. Each object contains the name of the body area and the view of the radiograph, the time of day when it was taken, the location of storage of the image in the system (file server, local hard disk, or RAM disk), and the examination number the image is part of. ImageFolder objects may have more than one representation (Fig 9), appearing either on view boxes of the control monitor, on view boxes of the image monitor, or on a body area list of the

\begin{tabular}{|c|}
\hline name of body area \\
\hline name of view \\
\hline time of day film was taken \\
\hline location of image in system \\
\hline examination number \\
\hline
\end{tabular}

Fig 8. ImageFolder object. 


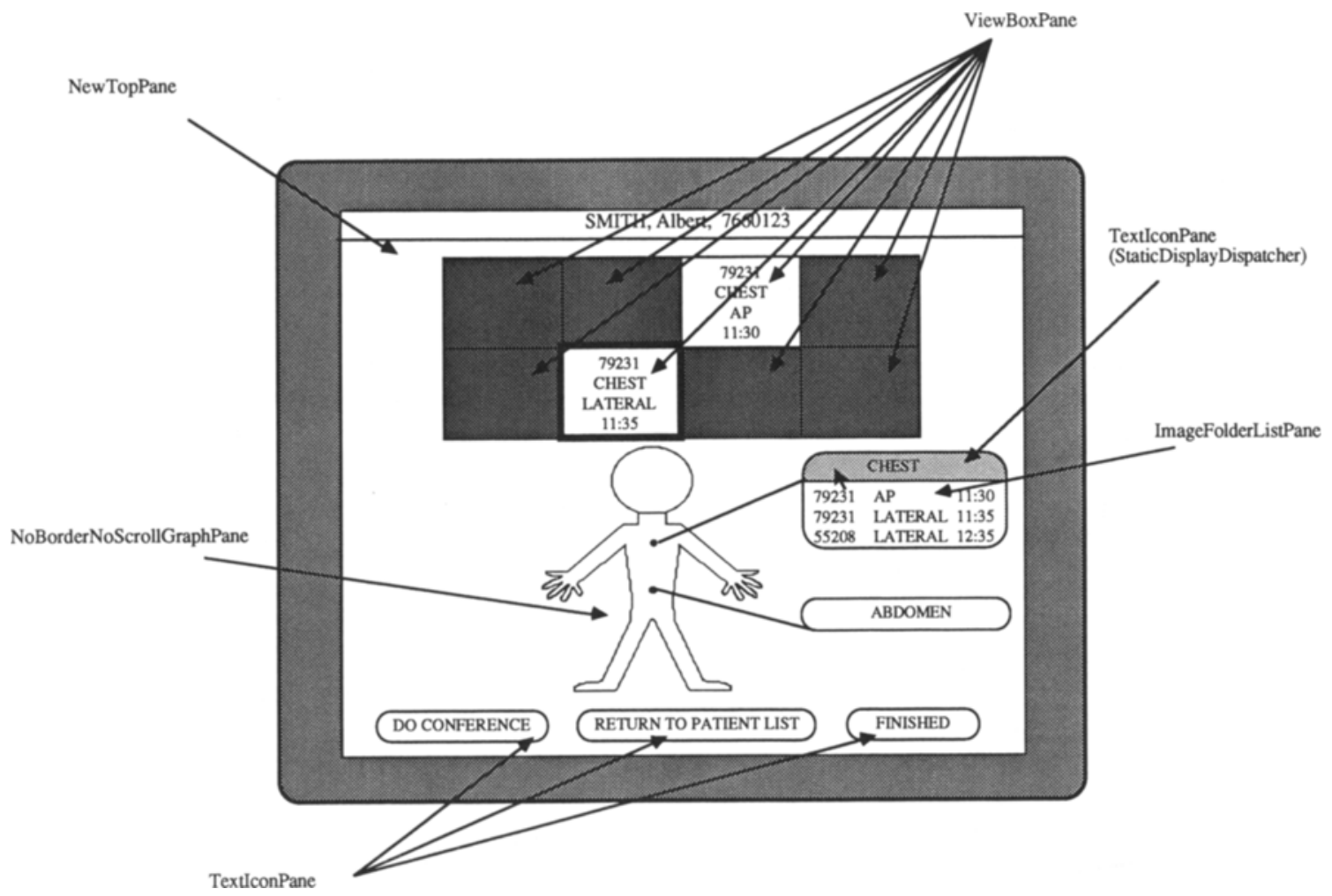

Fig 9. PatientExaminer model.

control monitor. A user may than create as many different views as necessary for the same ImageFolder object.

The PatientExaminer object (software developed by the author) is the model that manages the functions handled on the control monitor (Fig 9). It is responsible for the organization of the image labels and supports their placement on the viewboxes. The ViewBoxNavigator (software developed by the author) is the model that manages the embedded viewboxes of the image monitor (Fig 6).

When the PatientExaminer model is created, all ImageFolder instances of the patient are then linked as dependents to the model. Only the ImageFolders of images placed on viewboxes by the radiologist are linked as dependents to the ViewBoxNavigator model. Hence, through the use of dependency relationships, both models operate on the same ImageFolder objects without the need for duplication. The consistency of the ImageFolder objects is then maintained (eg, if an image is displayed from the ViewBoxNavigator, the PatientExaminer will be able to reflect that fact).
We emphasize that there are two models, one for each monitor, that remain coherent by exchanging messages upon changes initiated by the user (eg, selection of an image display). The user interface (including the image organization and navigation strategies) has been implemented using Smalltalk (Digitalk, Inc, Los Angeles, CA), an object-oriented programming language. ${ }^{14} \mathrm{The}$ image processing functions have been implemented in $\mathrm{C}$ and linked to the core of the user interface as Smalltalk/V primitives.

\section{FIELD TEST EVALUATIONS}

To evaluate the anthropomorphic organization and soft viewbox representation, radiologists and emergentologists at the Ottawa Civic Hospital were invited to participate in a series of supervised tests which followed the recommendations of Tombaugh et al. ${ }^{15}$ The objectives of the series of tests were (1) to determine the appropriateness of the anthropomorphic image organization strategy, (2) to determine the success of the soft view-box-based image navigation strategy, (3) to assess differences in user interface requirements 
and preferences between radiologists and emergentologists, and (4) to verify the efficacy and satisfaction with some related issues (eg, mouse sensitivity, visual feedback). Emphasis in the usability tests was placed on subjective measures of satisfaction and preference rather than on user performance. User performance was not measured because the users were instructed and guided as they worked with the system rather than left to explore on their own.

\section{Method}

\section{Participants}

Eleven radiologists and four emergentologists volunteered to serve in the experiment. The radiologists ranged in experience from residents to highly experienced radiologists with radiological subspecialties. Five participants had prior experience with computers, of which three had used a mouse.

\section{Design}

Because there was such a diversity in computer use and knowledge in the sample of participants, all subjects were required to run through a pretraining session with a mouse to learn basic skills in moving, selecting, dragging, and scrolling.

The appropriateness of the anthropomorphic image organization strategy (Fig 9) was assessed by comparing it with a "linear" organization strategy (Fig 10). The anthropomorphic strategy was designed to present a number of distinctive sublists of radiographs sorted by body parts and thus to give some visual clues about the content of the patient file. The linear organization simply presented all of the films in a single scrolling list. The soft view boxes were used for both linear and anthropomorphic conditions.

Each participant used both organization strategies. Half of the participants used the linear

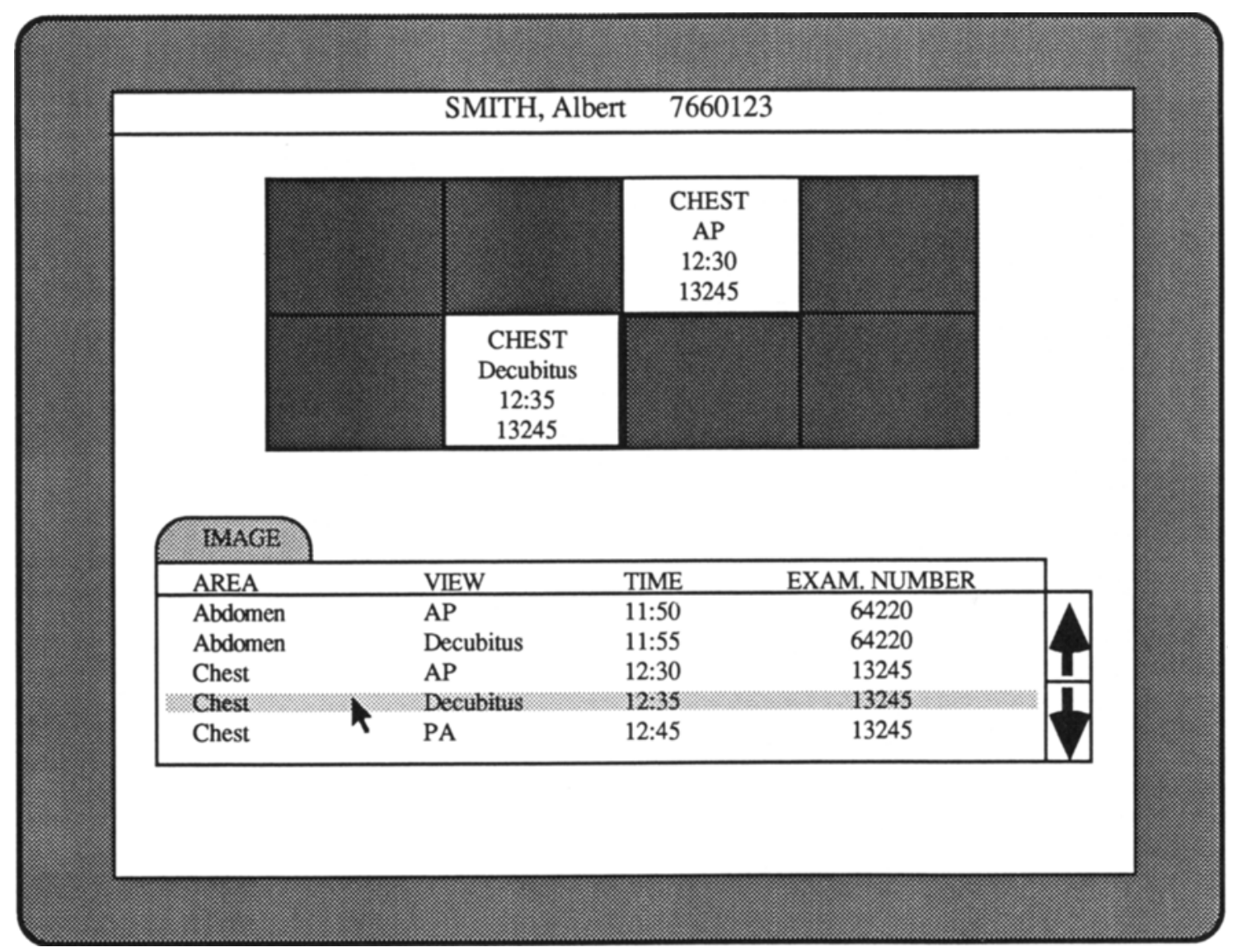

Fig 10. Linear organization strategy. 


\section{Pass the pointer through the numbers in the indicated order}

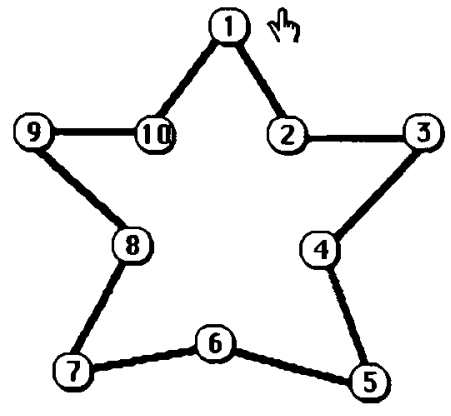

Nest

Fig 11. "Mouse-moving" practice screen.

strategy and half used the anthropomorphic strategy first; they then switched for the other half of the session. Separating the sample into two counterbalanced groups controlled for the possibility of confounding the preferences of each of the strategies with the order in which the strategies were presented.

\section{Materials}

A pretraining package to teach mousing skills was prepared using HyperCard on the Apple Macintosh computer (Apple Computer, Inc, $\mathrm{Cu}$ pertino, CA), for teaching moving (Fig 11), clicking (Fig 12), dragging (Fig 13), and scrolling (Fig 14). The interactive techniques demon- strated in this program were the same as those implemented on the work station.

Twenty radiographic images (acquired by a Konica laser film scanner, Konica Corp, Tokyo, Japan) were used in the experiment. These were sorted into five different body areas for the anthropomorphic organization: chest, cervical spine, cervical spine (myelogram), lumbar spine, and lumbar spine (myelogram). When the images were presented in the linear organization, the scrolling function was used because the linear list could display at most eight items at once. Specially designed scripts and questionnaires were developed for the test sessions. ${ }^{15}$ A sample of this script is shown in Table 1.

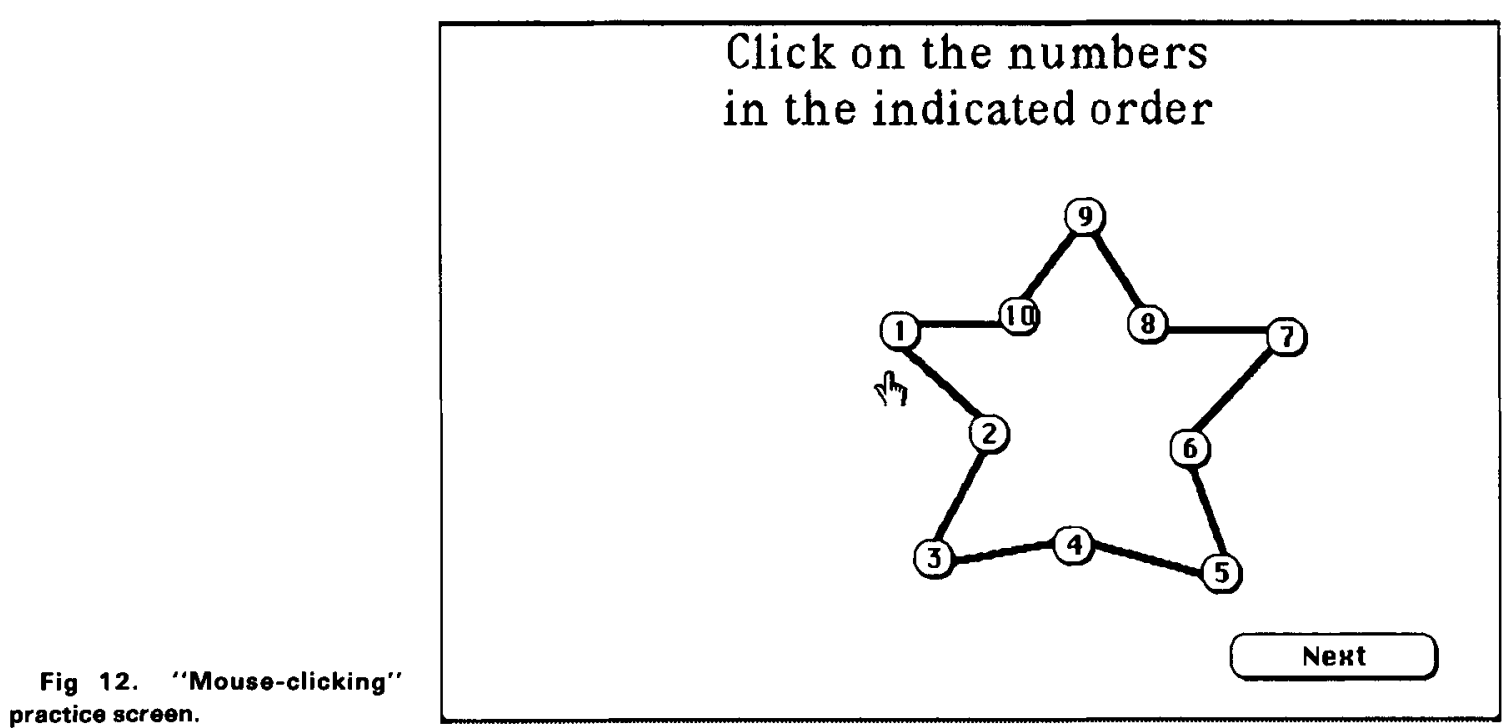


Fig 13. "Mouse-dragging" Drag the 6 buttons in to the correct locations

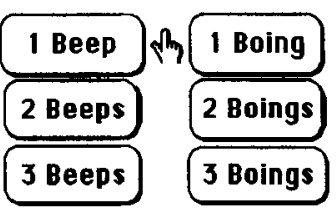

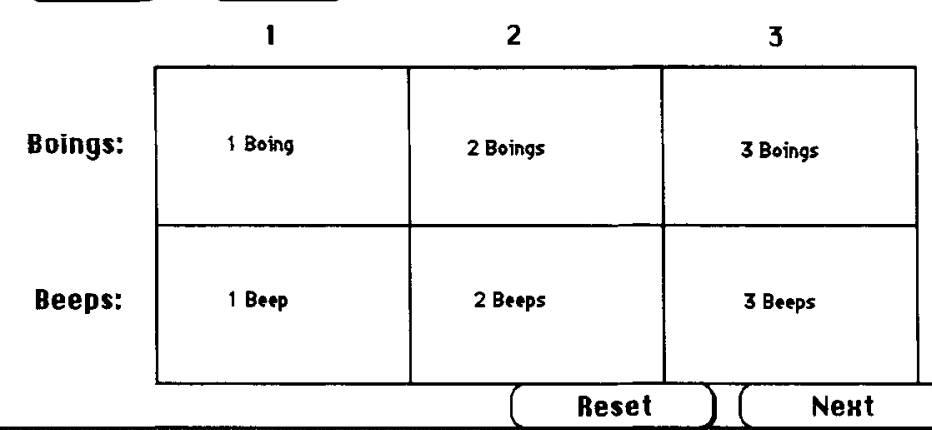

\section{Procedure}

An experimenter controlled the learning sessions following the script. The experiment was divided into two parts, mouse training, conducted using a Macintosh computer and user testing, conducted with the prototype system.

Mouse training. Each mousing skill was repeated by the subject until sufficient confidence and proficiency were shown. The experimenter was responsible for assessing the level of confidence and proficiency reached by the subject, and determining when the competence level was sufficient to move to the next skill.

Work station tests. Following the mouse practice, the subject sat at the work station and observed as the experimenter demonstrated the user interface. Half of the participants were presented with the anthropomorphic strategy first, while the other half were presented with the linear strategy first. Following the demonstration with one strategy the subject performed each of the functions, always remaining under the direction of the experimenter (Table 1). While the subject used the system any questions that arose were noted, and errors and hesitations were logged. Immediately following the use of the system with one strategy the user responded to a series of questions from a formal questionnaire, which queried acceptability and usefulness of specific functions. The process was then repeated

\begin{tabular}{|c|c|c|c|}
\hline \multicolumn{4}{|c|}{$\begin{array}{l}\text { Click arrows to scroll through exchange } \\
\text { Questions to answer } \quad \text { Foreign exchange }\end{array}$} \\
\hline $\begin{array}{l}\text { What country is last on the list? } \\
\text { What is the French franc worth? } \\
\text { Is the China erchange on the list? } \\
\text { How do the U.K. and Irish pounds } \\
\text { compore? } \\
\text { Is Turkey in the list? }\end{array}$ & \begin{tabular}{|l|} 
Austrolia dollor \\
Austria schilling \\
Bahamas dollar \\
Barbados dollor \\
Brazil cruzado \\
China renminbi \\
Colombia peso \\
Denmerk krone \\
Fiji dollor \\
France frone \\
Germany merk \\
Greece drachma \\
HongKong dollar \\
Hungary forint \\
India rupee \\
Irag dinar
\end{tabular} & $\begin{array}{l}.9360 \\
.1041 \\
1.2311 \\
.6098 \\
.0089 \\
.3308 \\
.0043 \\
.1909 \\
.8630 \\
.2160 \\
.7335 \\
.0091 \\
.1575 \\
.0246 \\
.0932 \\
3.9611 \\
\end{array}$ & \\
\hline Neнt & & & \\
\hline
\end{tabular}

Fig 14. "Scrolling" practice screon. 
Table 1. Part of a Script Used in Testing With the Linear Organization

1. (Experimenter demonstrates the system using a script which is not included in this table.)

This concludes my demonstration. Do you have any questions? What we'll do now is repeat the whole thing, but this time you'll be manipulating the mouse.

2. Start on "select a patient" screen.

First, show me the list of images for Mike Smith.

3. Linear organization screen comes up.

Display the oblique view of the cervical spine. (No scrolling required.)

Now display the lateral chest view. (Scrolling required.) Now put the lumbar spine, lateral view into this viewbox. (No scrolling required. Point to second viewbox.) Now, please put the first oblique view of the cervical spine into this viewbox. (Scrolling up required. Point to the first viewbox.)

And the last lumbar spine (myelo) view into that other viewbox. (Scrolling down required. Point to the sixth viewbox.)

Let's move the lumbar spine $x$-ray from this viewbox to that one. (Point from the first viewbox to the fourth viewbox.)

Now let's view the lumbar spine $x$-ray.

NOTE. Italics denote experimenter notes that are not read to the subjects.

with the second strategy. A final set of questions comparing the two types of organization was presented after the user had tried both types of organization. The average duration of the mouse training and experiment combined was 45 minutes.

\section{Results and Discussion}

The questionnaire emphasized preference and satisfaction information with a series of fivepoint rating scales. Results are presented in terms of separate mean ratings for the 11 radiologists and four emergentologists who participated in the study. The satisfaction ratings ranged from 1 ("very easy" or "not at all a problem") to 5 ("very difficult" or "very much a problem"). The usability goals adopted for these ratings was that a mean rating less than 2.0 would be considered satisfactory, whereas ratings of 2.0 or greater would be taken as an indication that design improvements should be considered. The choice of a stringent usability goal is appropriate considering the generally positive reaction to the system.

\section{Linear Versus Anthropomorphic Organization}

One of the primary objectives of the usability test was to compare participants' reactions to linear versus anthropomorphic strategies. Table 2 shows mean ratings for three questions that were asked separately for the linear and anthropomorphic organization. A mean rating of 2.0 or higher was interpreted to indicate a problem that should be improved.

Table 2 shows that all 15 participants indicated that using the anthropomorphic technique was not at all a problem-the highest rating possible. In contrast, mean ratings for overall difficulty using the linear strategy were an unacceptable 2.9 for radiologists and a more acceptable 1.5 for emergentologists. The two techniques were rated essentially the same (and acceptable) in terms of the difficulty of finding an image (mean ratings, 1.2 to 1.5 ). Difficulty getting an overview of the images in the file was borderline acceptable (mean ratings, 1.7 and 1.8) for both techniques by radiologists and emergentologists, except that the linear organization received a mean rating by radiologists $(2.8)$ that indicated that improvements are desirable.

The apparent preference of the radiologists for the anthropomorphic strategy, shown in Table 2, was confirmed with four questions that asked participants to rate directly their preference for the linear versus anthropomorphic techniques on scales where 1 indicated a preference for the anthropomorphic organization, 5 a preference for the linear organization, and 3 no preference. Table 3 shows that the four emergentologists had little preference in terms of ease of mouse use, ease of locating images, ease of obtaining an overview of file content, and ease to set up

Table 2. Mean Ratings of Linear and Anthropomorphic Strategies

\begin{tabular}{lcccc}
\hline \multicolumn{1}{c}{ Question } & \multicolumn{2}{c}{ Anthropomorphic } & \multicolumn{2}{c}{ Linear } \\
\cline { 2 - 5 } & $\begin{array}{c}\text { Radiologists } \\
(n=11)\end{array}$ & $\begin{array}{c}\text { Emergentologists } \\
(n=4)\end{array}$ & $\begin{array}{c}\text { Radiologists } \\
(n=11)\end{array}$ & $\begin{array}{c}\text { Emergentologists } \\
(n=4)\end{array}$ \\
\hline Using the organization strategy & 1.0 & 1.0 & 2.9 & 2.8 \\
Getting an overview of images & 1.8 & 1.8 & 1.5 \\
Finding an image & 1.2 & 1.5 & 1.8 & 1.5 \\
\hline
\end{tabular}

NOTE. Ratings: 1 , not at all a problem; 5 , very much a problem. 
Table 3. Mean Preference Ratings for Linear or Anthropomorphic Organizations

\begin{tabular}{lcc}
\hline Question & $\begin{array}{c}\text { Radiologists } \\
(\mathrm{n}=11)\end{array}$ & $\begin{array}{c}\text { Emergentologists } \\
(\mathrm{n}=4)\end{array}$ \\
\hline $\begin{array}{l}\text { Using the organization } \\
\text { strategy }\end{array}$ & 3.7 & 2.8 \\
Getting an overview & & \\
of images & 3.8 & 2.5 \\
Finding an image & 3.8 & 2.8 \\
Using the mouse & 3.0 & 2.5 \\
\hline
\end{tabular}

NOTE. Ratings: 1, much prefer linear; 3, no preference; 5 , much prefer anthropomorphic.

view boxes (mean ratings, 2.5 to 2.8 ). Radiologists showed no preference in ease of setting up view boxes (mean, 3.0 and 9 of 11 radiologists showing no preference), but some preferences for the ease of using the anthropomorphic organization for mouse use, locating images, and getting an overview of the file (means, 3.7 and 3.8). In fact, 8 of the 11 radiologists preferred the anthropomorphic list for locating images, 7 preferred it for getting a file overview, and 6 preferred it for using the mouse.

The finding that radiologists slightly preferred the anthropomorphic organization whereas emergentologists were more indifferent probably stems from the size of files that the two groups usually process. Radiologists tend to use complete patient files, which often include multiple examinations, whereas emergentologists tend to see a single examination based on the problem that brought the individual to the emergency room. One question asked in the questionnaire was the frequency with which physicians might view more than 10 films for a given body area. All emergentologists indicated that they almost never handle more than 10 films for one area, whereas all but one radiologists reported that they sometimes handle more than 10 films for a given body area. Thus, radiologists see the need for the hierarchical organization provided by the anthropomorphic strategy, whereas emergentologists find the linear organization to be satisfactory.

Whether the preference of the radiologists for the anthropomorphic list result from the anthropomorphic organization per se or from the use of hierarchical organization as compared with linear organization cannot be determined from this experiment. However, it is clear that some kind of hierarchical organizational help is required for radiologists, and that they find the anthropomorphic organization useful.
Although comparison between the two image list organizations was possible on the issues discussed previously, there were also special issues associated with each type of list. In particular, the linear lists required scrolling through the image labels whereas the anthropomorphic lists required hierarchical selection. Table 3 deals with the special issues associated with each organizational strategy.

Linear strategy issues. Because all 20 images could not be displayed simultaneously in the linear list, scrolling was necessary. Three questions probed the difficulty of scrolling in the linear list. Results of the difficulty ratings for the linear organization strategy are presented in the first section of Table 4. Forgetting that the list had more items than those displayed, and scrolling through the list in the wrong direction were not problems (means, 1.3 to 1.6 ). Radiologists (mean, 3.0) and to lesser extent emergentologists (mean, 1.8) considered it a problem to scroll through the list of items. The negative reaction by radiologists was due to the fact that a mouse button click was required for each new item displayed. An easier scrolling technique, eg, a continuous scrolling capability while the mouse button was held down, was intentionally omitted as too complex for novice computer users. Radiologists, who see film retrieval as a major part of their work and who would use the work station regularly, reacted more strongly to this inefficiency than did emergentologists. A continuous scrolling technique will be available in future versions and will be required with the anthropo-

Table 4. Mean Ratings of Features Specific to the Linear and Anthropomorphic Lists

\begin{tabular}{|c|c|c|}
\hline Question & $\begin{array}{l}\text { Radiologists } \\
\qquad(\mathrm{n}=11)\end{array}$ & $\begin{array}{l}\text { Emergentologists } \\
\qquad(n=4)\end{array}$ \\
\hline \multicolumn{3}{|l|}{ Linear organization } \\
\hline \multicolumn{3}{|c|}{ Forgetting the list had more } \\
\hline items & 1.5 & 1.3 \\
\hline \multicolumn{3}{|c|}{ Moving the list the wrong } \\
\hline way & 1.6 & 1.5 \\
\hline $\begin{array}{l}\text { Only being able to } \mathrm{m} \\
\text { the list one item a }\end{array}$ & & \\
\hline time & 3.0 & 1.8 \\
\hline \multicolumn{3}{|c|}{ Anthropomorphic organization } \\
\hline \multicolumn{3}{|c|}{$\begin{array}{l}\text { Having to choose twice to } \\
\text { select an image (body }\end{array}$} \\
\hline part, then image) & 2.1 & 2.0 \\
\hline \multicolumn{3}{|c|}{ Selecting the wrong cate- } \\
\hline
\end{tabular}


morphic organization, as well as the linear organization, because of the finding that a large proportion of the radiologists frequently use more than 10 images per body area.

Anthropomorphic organization issues. Whereas the linear list strategy required scrolling to view all images, the anthropomorphic strategy required a two-level, hierarchical selection process. When the body area was selected, the list of images for that area was displayed. The desired images were then selected from this list. To view more than one image for a given body part, the body part had to be reselected before selecting the image. Two questions in the second section of Table 4 probed the rated difficulty of this selection process. The possibility of selecting the wrong body part was not considered a problem by either radiologists (mean, 1.1) or emergentologists (mean, 1.3). However, the two-level selection procedure was considered borderline unacceptable (mean for radiologists, 2.1; mean for emergentologists, 2.0). This suggests that a procedure in which repeated selections of images for the same body part, without reselecting the body part, should be implemented and evaluated. It is not clear if the dissatisfaction with hierarchical choice would be eliminated by this change, but it seems feasible, particularly because radiologists will often view all images for a particular body area before moving on to the next body area.

\section{View Boxes}

The second major objective of the usability study was to get participants' initial impressions of the usability of the view-box metaphor for image navigation. If some physical representation of an organized subset of images is not provided on the screen, then physicians will either have to maintain the organization in memory or write it on paper. Three questions were concerned with usage of the view boxes. Ratings were on a scale of 1 to 5 , where 1 meant "very easy" and 5 meant "very difficult".

Table 5 shows that participants judged that it was easy to drag image information from a list to a view box (means, 1.8 and 1.0), drag image information from a view box to another view box (means, 1.5 and 1.0), and pop-up the set of view boxes on the image monitor (means, 1.4 and 1.0). In addition, two thirds of the participants thought
Table 5. Mean Ratings of Features Specific to Use of the Viewboxes

\begin{tabular}{lcc}
\hline \multicolumn{1}{c}{ Question } & $\begin{array}{c}\text { Radiologists } \\
(\mathrm{n}=11)\end{array}$ & $\begin{array}{c}\text { Emergentologists } \\
(\mathrm{n}=4)\end{array}$ \\
\hline $\begin{array}{l}\text { Dragging image label from list } \\
\text { to viewbox }\end{array}$ & 1.8 & 1.0 \\
$\begin{array}{l}\text { Moving the list the wrong way } \\
\text { Only being able to move the } \\
\quad \text { list one item at a time }\end{array}$ & 1.5 & 1.0 \\
$\begin{array}{l}\text { Displaying viewboxes on im- } \\
\quad \text { age monitor }\end{array}$ & 1.4 & 1.0 \\
\hline NOTE Ratings: 1, not at all a problem:5. very much a problem
\end{tabular}

the information shown in a view box was satisfactory, and 13 of 15 participants believed the information in the view boxes was properly organized.

These results indicate that the view box metaphor may provide a convenient means to organize a subset of images and navigate through them. The view-box metaphor is particularly appealing because it capitalizes on an effective strategy that is already well known to radiologists and other physicians who read roentgenograms.

Although the physicians liked the view-box analogy, further tests are required to determine if it will be an aid to them in their work. The goals of this experiment were to determine whether or not the physicians could learn to use the system and whether they found it satisfactory. The physicians were not using the system to make actual diagnoses and, consequently, were not developing viewing strategies. In further testing planned for this system, radiologists will report on actual patient files, using their own viewing strategies. It is in this type of realistic use that the value of the view boxes will be determined. If the view-box metaphor is not an aid to organization and planning strategy, it is expected that the physicians will bypass the extra step of dragging the images to the viewboxes and will select images for viewing directly from the list of roentgenograms.

\section{Mouse, Monitor, and Cursor}

A tertiary purpose of the usability tests was to evaluate a variety of features of the graphic interface environment. A series of questions were designed to capture the impressions of the participants on various issues such as using the mouse, the monitors, and the cursor. Ratings were on a scale of 1 to 5 , where 1 meant "very easy" and 5 meant "very difficult". 
The results shown in Table 6 indicate a high level of acceptance of features with three exceptions. Moving the mouse from screen to screen (mean, 1.9), locating the cursor after a screen change (mean, 2.9), and locating the cursor on the image screen (mean, 2.4) were rated lower than other issues in Table 6.

Visibility of the cursor on the image screen was a problem and was easily improved. A black cursor with a narrow white line around it is visible against a background of any shade of grey from black to white. If the black part of the cursor is not visible, the white line is, and vice versa. There is no way to make it more natural to move the cursor between the screens, although the negative reactions may stem from the difficult in locating the cursor rather than the movement required.

\section{Summary}

Requirements for image organization and navigation were identified. A prototype was implemented. A carefully scripted test was performed with 11 radiologists and four emergentologists. The main objectives of this test were to determine ease of use of the system, determine the appropriateness of the prototype anthropomorphic image organization strategy compared with

Table 6. Mean Ratings of Other Interface Design Features

\begin{tabular}{|c|c|c|}
\hline Question & $\begin{array}{c}\text { Radiologists } \\
(n=11)\end{array}$ & $\begin{array}{l}\text { Emergentologists } \\
\qquad(n=4)\end{array}$ \\
\hline $\begin{array}{l}\text { Moving the mouse: control } \\
\text { screen }\end{array}$ & 1.2 & 1.3 \\
\hline $\begin{array}{l}\text { Moving the mouse: image } \\
\text { screen }\end{array}$ & 1.6 & 1.3 \\
\hline $\begin{array}{l}\text { Moving the mouse from } \\
\text { screen to screen }\end{array}$ & 1.8 & 2.0 \\
\hline $\begin{array}{l}\text { Locating the cursor on the } \\
\text { control screen }\end{array}$ & 1.4 & 1.3 \\
\hline $\begin{array}{l}\text { Locating the cursor on the } \\
\text { image screen }\end{array}$ & 2.5 & 2.3 \\
\hline $\begin{array}{l}\text { Locating the cursor after a } \\
\text { screen change }\end{array}$ & 2.9 & 2.8 \\
\hline Displaying an image & 1.3 & 1.0 \\
\hline Clicking to select an image & 1.7 & 1.3 \\
\hline $\begin{array}{l}\text { Knowing what image is se- } \\
\text { lected for display }\end{array}$ & 1.2 & 1.3 \\
\hline Reading the control screen & 1.2 & 1.0 \\
\hline $\begin{array}{l}\text { Size of characters on the con- } \\
\text { trol screen }\end{array}$ & 1.1 & 1.0 \\
\hline Clutter of the control screen & 1.2 & 1.3 \\
\hline $\begin{array}{l}\text { Information adequacy on the } \\
\text { control screen }\end{array}$ & 1.2 & 1.0 \\
\hline
\end{tabular}

NOTE. Ratings: 1 , not at all a problem; 5 , very much a problem. a more conventional linear strategy for organizing the images in patient files, and to determine initial reaction of the soft view-box-based image navigation strategy.

Generally, the test indicated that the interface was successful. Both the radiologists and emergentologists were able to use the features and felt satisfied with the way in which the system worked. Given a small amount of training, the physicians were able to use the mouse to select and locate images without difficulty.

In terms of functional mechanics, both image organization strategies were rated easy to use. Easily corrected problems were identified with single-item scrolling and the selection process for viewing more than one image for a given body area in the anthropomorphic list. No preference for one strategy emerged from the emergentologists, but radiologists expressed a preference for the anthropomorphic strategy. This may be explained by the more complex nature of the radiologists' tasks and experience with larger sets of examinations, which may require a more sophisticated strategy for presenting the image content of a patient file.

In terms of usability, the soft view boxes were thought to present no problems and were considered easy to use. Moving the cursor from one screen to the other and finding the cursor were problematic and were improved.

\section{CONCLUSION}

Our proposed digital image organization strategy is based on an anthropomorphic organization (Fig 3). The center of the control monitor is occupied by the sketch of a human body around which labels of images referring to the same body area are grouped. This graphic index of images provides an overview of the image content of the patient folder. Our image navigation strategy is based on a soft representation of the physical view boxes (Fig 5). It mimics the spatial arrangement in the analog world (onto physical view boxes). The soft view-box surface constitutes a bidimensional space on which the user arranges radiographs represented by icons. Although our PACS workstation has only one monitor for displaying images, the resulting visual workspace is augmented by this strategy.

Our image organization and navigation strategies were implemented in Smalltalk/V, an object- 
oriented programming language, and evaluated in a series of tests at the Ottawa Civic Hospital. Radiologists did prefer the anthropomorphic organization to a simple list-type organization used for comparison. The soft view boxes were found to be of theoretical interest for image navigation but their actual usefulness remains to be experimentally assessed. Based on initial comments and suggestions, the following features seem useful: (1) automatic loading of the view boxes with all views of an examination, in which the order of the views could be determined by the nature of the examination and by user preferences (most probably to be handled by an expert system); (2) automatic sequencing of the display of the views planned on the view boxes (eg, provision of a pair of "next/previous" buttons that would display images in sequence from left to right and top to bottom); (3) provision of multiple planes of soft viewboxes (to create a three-dimensional space) for cross-examination comparison of corresponding views; and (4) provision of a larger two-dimensional view-box space (larger than the traditional $4 \times 2$ grid) for examinations with large number of views.

\section{ACKNOWLEDGMENT}

The authors wish to acknowledge the radiologists, emergentologists, and personnel of the Ottawa Civic Hospital who generously donated their time to our experiments. Also special thanks are given to Marjorie Coristine, Dr J.W. Tombaugh, and Cynthia Houston for their considerable assistance in the preparation and realization of the field tests.

\section{REFERENCES}

1. Huang HK: Elements of Digital Radiology. Englewood Cliffs, NJ, Prentice-Hall, 1987

2. Horii SC, Isles G, Bergeron RT: Display preferences for viewing CT scans. Society of Photo-Optical Instrumentation Engineers Medicine XIV/PACS IV, vol 626:1986, pp 426429

3. Johnston RE, Rogers DC, Creasy JL, et al: How to evaluate a medical imaging display workstation. Society of Photo-Optical Instrumentation Engineers Medical Imaging, vol 767:1987, pp 616-621

4. Kasday LR: Human factors considerations in PACS design. SPIE Medicine XIV/PACS IV, vol 626:1986, pp $581-592$

5. McNeill KM, Fisher HD: A model for radiologic workstation user interface design. Society of Photo-Optical Instrumentation Engineers Medical Imaging, vol 767:1987, pp 713-716

6. Rogers DC, Johnston RE, Brenton B, et al: Predicting PACS console requirements from radiologists' reading habits. Society of Photo-Optical Instrumentation Engineers Picture Arch PACS III for Medical Applications, vol 536:1985, pp 88-95

7. Schuttenbeld HHW, Romeny B: Design of a userinterface for a PACS viewing station. SPIE Medical Imaging, vol 767:1987, pp 844-848

8. Vercillo R, Fisher HD, Lamoreaux RD, et al: Digital image review console. Society of Photo-Optical Instrumentation Engineers Medical Imaging, vol 767:1987, pp 708-712

9. Taira RK: Operational characteristics of pediatric radiology: Image display stations. Society of Photo-Optical Instrumentation Engineers Medical Imaging, vol 767:1987, pp 571.576

10. Chang SK: Image information systems. Proceedings of the Institute of Electrical and Electronic Engineers, vol 73:1985, pp 754-764

11. Wilson GA, Herot CF: Semantics vs graphics-To show or not to show. Proceedings of the Sixth Institute of Electrical and Electronic Engineers International Conference on Very Large Data Bases. 1980, pp 183-196

12. O'Malley KG, Giunta JA: The alternator: Determination of its fundamental features, as a basis for design of a PACS diagnostic workstation. Medical Imaging II, vol 914: 1988, pp 988-994

13. Shneiderman B: Designing the user interface: Strategies for effective human-computer interaction. Reading, MA, Addison-Wesley, 1987

14. Smalltalk/V Tutorial and Programming Handbook. Los Angeles, CA, Digitalk, Inc, 1986

15. Tombaugh JW, Dillon RF, Coristine M: Goal setting and user testing to ensure a PACS interface satisfactory to radiologists. Society of Photo-Optical Instrumentation Engineers Medical Imaging III, 1093, 1989 